\title{
Electrical transport through individual DNA molecules
}

\author{
Xin-Qi Li and YiJing Yan \\ Department of Chemistry, Hong Kong University of Science and Technology, Kowloon, \\ Hong Kong
}

(March 30, 2001)

\begin{abstract}
A theoretical study is presented to quantitatively analyze the transport experiment through individual DNA molecules reported recently by Porath et al. [Nature 403, 635 (2000)]. A variety of valuable quantities are identified by contacting the theoretical model with the measured data. The partially decoherent nature on the GC pairs of DNA is elaborated in contrast to the completely incoherent hopping mechanism discussed in the context of charge transfer experiments.
\end{abstract}

PACS numbers: 72.10.-d,72.90.+y

Typeset using REVTEX 
DNA has the special double-helix structure with $\pi$-electron cores of well stacking bases, which may provide an one-dimensional pathway for charge transport. Since first posed in 1960s, the question whether or not DNA is able to conduct electrical charges is still debated. Due to the advent of molecular electronics as well as other biological considerations, this issue has stimulated intense research interest. Recent studies proposed that DNA seems to be one of the most promising candidates in the application of functional nano-electronic devices [1]. Very recently, direct measurements for the electrical transport through DNA molecules were performed [2,3]. In particular, Porath et al. [3] measured the electrical transport through the individual DNA molecules. The observed nonlinear current-voltage (I-V) characteristics clearly suggest that DNA molecules be good molecular semiconductors. In this letter, we carry out a quantitative analysis for the experimental I-V characteristics [3], and discuss the relevant physical implications.

The theoretical model for the experimental setup is schematically shown in Fig. 1. In the experiment [3], the DNA sample of $10.4 \mathrm{~nm}$-long chain containing 30 GC pairs was studied. The ab initio calculation [4] showed a large HOMO-LUMO gap (10.07 eV) of the single GC pair. We thus adopt a homogeneous one-band tight-binding model for the HOMOmediated charge transport through the DNA molecules under study. To proceed, consider the following Hamiltonian to describe the system in Fig. 1:

$$
\begin{aligned}
H & =H_{0}+H_{I}, \\
H_{0} & =H_{M}+H_{L}+H_{R}+H_{\text {res }} .
\end{aligned}
$$

Here $H_{0}$ describes the separated subsystems of the DNA molecule $\left(H_{M}\right)$, the left and right metal electrodes $\left(H_{L}\right.$ and $\left.H_{R}\right)$, and the dephasing reservoirs $\left(H_{\text {res }}\right) . H_{I}$ couples the DNA molecule to the electrodes and the dephasing reservoirs. Following Büttiker's idea for the inelastic scattering effect [5, 6], the phase-breaking processes on the GC-pairs are modeled by coupling each GC-pair to a fictitious electronic reservoir, see Fig. 1. The dephasing strength is characterized by the coupling parameter $\eta$.

To carry out the nonlinear transport characteristics, the knowledge of the energy- 
dependent transmission coefficient through the molecule is required. Owing to the open boundary conditions associated with the voltage electrodes and dephasing reservoirs, the Green's function method would be the proper and versatile theoretical tool. We thus introduce the following Green's function

$$
\mathcal{G}(E)=\left(E-\mathcal{H}_{\mathrm{eff}}\right)^{-1}
$$

where $\mathcal{H}_{\text {eff }}$ is the effective Hamiltonian of the reduced molecular system, obtained by eliminating the degrees of freedom of the two electrodes and the dephasing reservoirs. As a simple model, the electrodes and dephasing reservoirs can be described by semi-infinite one-dimensional tight-binding chains [6], which result in the self-energy corrections to the Hamiltonian of the molecule as

$$
\mathcal{H}_{\mathrm{eff}}=H_{M}+\Sigma_{L}|1\rangle\left\langle 1\left|+\Sigma_{R}\right| N\right\rangle\left\langle N\left|+\sum_{j=1}^{N} \Sigma_{j}\right| j\right\rangle\langle j|
$$

$\Sigma_{L(R)}$ and $\Sigma_{j}$ are respectively the self-energies resulting from the coupling of the molecule to the left (right) electrodes, and the $j$ th dephasing reservoir. Using Dyson equation, the self-energy $\Sigma_{\mu}(\mu=L, R$, and $\{j\})$ can be carried out as

$$
\Sigma_{\mu}=\frac{V_{\mu}^{2}}{E-E_{\mu}-\sigma_{\mu}},
$$

where $\sigma_{\mu}$ is the self-energy correction of the semi-infinite chain to the ending-site of the chain attached to the DNA molecule, and has the following expression [6]

$$
\sigma_{\mu}=\frac{E-E_{\mu}}{2}-i\left[\gamma_{\mu}^{2}-\left(\frac{E-E_{\mu}}{2}\right)^{2}\right]^{1 / 2}
$$

Here $V_{\mu}$ are the coupling strengths between the molecule and the electrodes/reservoirs: $V_{\mu}=V_{L(R)}$ if $\mu=L(R)$; and $V_{\mu}=\eta$ if $\mu=\{j\} . \quad E_{\mu}$ and $\gamma_{\mu}$ denote the site energies and the nearest-neighbor hopping strengths of the homogeneous tight-binding electrode and reservoir chains. We let $\gamma_{L(R)}=\gamma$ for the left (right) electrode and $\gamma_{j}=\gamma_{0}$ for the dephasing reservoirs. Thus, $4 \gamma$ and $4 \gamma_{0}$ characterize the band widths of the electrodes and the dephasing reservoirs, which are assumed in this work to be commonly $5 \mathrm{eV}$. 
To connect the Green's function with the transmission coefficients between any pair of reservoirs, say, from $\mu$ to $\nu$, consider a linear transport between them. It can be shown [7] that the linear conductance at low temperatures is $g_{\mu \nu}=\left(2 e^{2} / \pi \hbar\right) \Delta_{\mu} \Delta_{\nu}\left|\mathcal{G}_{\mu \nu}(E)\right|^{2}$. Here, $\Delta_{\mu}$ is associated with the self-energy by $\Delta_{\mu}=-\operatorname{Im} \Sigma_{\mu}$. $\mathcal{G}_{\mu \nu}(E)$ is the matrix element of $\mathcal{G}(E)$ between the molecular sites connecting with the $\mu$ th and $\nu$ th reservoirs $(\mu, \nu=L, R$, and $\{j\})$. On the other hand, the linear conductance is given by the Landauer-Büttiker formula [8] as $g_{\mu \nu}=\left(e^{2} / h\right) T_{\mu \nu}$. We therefore establish the following desired relationship between the transmission coefficient $T_{\mu \nu}$ and the Green's function:

$$
T_{\mu \nu}=4 \Delta_{\mu} \Delta_{\nu}\left|\mathcal{G}_{\mu \nu}(E)\right|^{2} .
$$

Note that in the wide band limit, $\Delta_{\mu} \simeq V_{\mu}^{2} / \gamma_{\mu}$. We thus conclude that in the special case $V_{\mu}=\gamma_{\mu}$, Eq. (6) reduces to the result of Refs. [6,9]. After knowing $T_{\mu \nu}$, we are ready to carry out the total effective transmission coefficient from the left electrode to the right one. We have recently shown that for each individual electron being emitted from the electrode, the effective transmission coefficient in the presence of phase-breaking is of the same form as that first derived by D'Amato and Pastawski [10,6]:

$$
\mathcal{T}_{\text {eff }}(E)=T_{L R}+\sum_{\mu, \nu=1}^{N} K_{\mu}^{(L)} W_{\mu \nu}^{-1} K_{\nu}^{(R)} .
$$

Here $K_{\mu}^{(L)}=T_{L \mu}, K_{\nu}^{(R)}=T_{\nu R} . W^{-1}$ is the inverse of the matrix $W$ defined in terms of the matrix elements $W_{\mu \nu}=\left(1-R_{\nu \nu}\right) \delta_{\mu \nu}-T_{\mu \nu}\left(1-\delta_{\mu \nu}\right)$, with $R_{\nu \nu}=1-\sum_{\mu(\neq \nu)} T_{\nu \mu}$. The physical meaning of Eq. (7) is clear: the first term is the coherent contribution of tunneling, whereas the second term denotes the incoherent component owing to electron suffering the dephasing processes.

With the knowledge of the transmission function $\mathcal{T}_{\text {eff }}(E)$, it is straightforward to evaluate the I-V characteristics by applying the standard formalism based on the scattering theory of transport [ []

$$
I=\frac{2 e}{h} \int_{-\infty}^{\infty} d E \mathcal{T}_{\text {eff }}(E)\left[f_{L}(E)-f_{R}(E)\right]
$$


Here $f_{L / R}(E)=\left\{\exp \left[\left(E-\mu_{L / R}\right) / k_{\mathrm{B}} T\right]+1\right\}^{-1}$ is the Fermi function. The room temperature $\left(k_{\mathrm{B}} T \simeq 26 \mathrm{meV}\right)$ will be considered in the numerical results, in order to keep consistency with the experiment [3]. $\mu_{L}$ and $\mu_{R}$ are the electrochemical potentials of the two metal electrodes, whose values depend on the applied bias voltage. Following Datta et al. [11, we set $\mu_{L}=E_{f}+(1-\kappa) e \mathcal{V}$, and $\mu_{R}=E_{f}-\kappa e \mathcal{V}$, where $E_{f}$ and $\mathcal{V}$ are respectively the equilibrium Fermi energy and the applied voltage. $\kappa$ is a useful parameter in characterizing how the applied voltage $\mathcal{V}$ is divided across the two junctions between the molecule and the electrodes.

Figure 2 shows the calculated I-V characteristics, together with the experimental data taken from Ref. [3]. Below we discuss how to make contact of the theoretical model with the measured data in order to get useful informations. Firstly, we notice the asymmetric gaps $V_{c}^{( \pm)}$under positive and negative bias voltages: $V_{c}^{(+)}=1.6 \mathrm{eV}$ and $V_{c}^{(-)}=0.8 \mathrm{eV}$. This asymmetry suggests $\kappa=1 / 3$, instead of $1 / 2$ as usual [11]. Secondly, the experimental differential conductance [3] showed a peak structure with the voltage spacings $\Delta \mathcal{V}$ of $0.1 \sim$ $0.5 \mathrm{eV}$. By taking the average value $\Delta \mathcal{V} \simeq 0.25 \mathrm{eV}$ and $\kappa=1 / 3$, we estimate the DNA molecular energy level spacing as $\Delta E=\kappa \Delta \mathcal{V} \simeq 0.08 \mathrm{eV}$. Since the DNA under study consists of 30 GC-pairs, from the simple consideration that the energy bandwidth $4 t=$ $30 \times 0.08=2.4 \mathrm{eV}$, we obtain the important information for the hoping strength between the nearest-neighbor GC pairs, $t \simeq 0.6 \mathrm{eV}$, which is in good agreement with the ab initio calculation [4. Thirdly, the equilibrium Fermi level $E_{F}$ of the electrodes can be estimated via $E_{F}-E_{G}=2 t+\kappa V_{c}^{(+)} \simeq 1.73 \mathrm{eV}$, where $E_{G}$ is the HOMO level of the single GC pair. This information would be helpful in understanding and predicting the voltage gaps in transport experiments. Finally, we notice the asymmetry of the entire profile of the experimental I-V curve under positive and negative bias voltages. We temporarily understand this feature by the distinct coupling strengths of the DNA molecule to the metal electrodes: $V_{L / R}=3 \mathrm{meV}$ for positive voltage, and $V_{L / R}=2 \mathrm{meV}$ for negative voltage. With these parameters, the theoretical results are in quantitative agreement with the measured data as shown in Figure 2 . 
Since the I-V characteristic is an integrated result of the transmission coefficient, the dephasing effect is not quite prominent on the I-V curve, except that the voltage gaps and the current steps due to the discrete molecular energy levels are smoothed by the phase-breaking scattering as shown in Fig. 2. However, it has drastic effect on the differential conductance as shown in Fig. 3. Roughly speaking, the dephasing would broaden the conductance peaks. More precisely, the differential conductance $d I / d V$ which corresponds to the transmission coefficient $\mathcal{T}_{\text {eff }}(E)$ at the specific voltage would change with the dephasing strength as representatively shown in the inset of Fig. 3. The turnover behavior has also been discovered in other context of tunneling with dissipation [10]. Note that the experimental peak width is of $\sim 0.2 \mathrm{eV}$, while its spacing $\sim 0.25 \mathrm{eV}$. It may thus suggest that the charge motion along the stacked GC-pairs is only partially dephased in the referred experiment [3]. It is worth emphasizing that this motional nature is essentially different from the hopping mechanism discussed in the context of long-range charge transfer phenomena in DNA [12], where the quantum coherence is regarded as being completely destroyed on the GC pairs. We may attribute this distinct nature to the different environments in which the DNA molecules located. Since the electronic dephasing would significantly influence the long-range charge transfer efficiency [10], we here suggest to further identify the dephasing strength on the GC pairs, by investigating the electrical transport through various sequence of DNA molecules and under different environments.

In summary, we have presented a theoretical formalism for the transport through DNA molecules. By making contact of the theoretical model with the experimental data, a variety of valuable quantities were identified, and the theoretical result was in quantitative agreement with experiment. The modest dephasing nature on the GC pairs was emphasized in particular, viewing its close relevance to the long-range charge transfer in DNA. Finally, the present theoretical formalism is quite general, which can also be employed to describe the transport through other molecular wires. 


\section{ACKNOWLEDGMENTS}

Support from the Research Grants Council of the Hong Kong Government and the National Natural Science Foundation of China is gratefully acknowledged. 


\section{REFERENCES}

[1] C. A. Mirkin, R. L. Letsinger, R. C. Mucic, and J. J. Storhoff, Nature (London) 382, 607 (1996); A. P. Alivisatos, K. P. Johnson, T. E. Wilson, C. J. Loveth, M. P. Bruchez, and P. G. Schultz, Nature (London) 382, 609 (1996); E. Braun, Y. Eichen, U. Sivan, and G. Ben-Yoseph, Nature (London) 391, 775 (1998); E. Winfree, F. Liu, L. A. Wenzler, and N. C. Seeman, Nature (London) 394, 539 (1998); C. D. Mao, W. Q. Sun, Z. Y. Shen, and N. C. Seeman, Nature (London) 397, 144 (1999); Y. Okata, T. Kobayashi, K. Tanaka, and M. Shimomura, J. Am. Chem. Soc. 120, 6165 (1998).

[2] H-W. Fink and C. Schönenberger, Nature 398, 407 (1999).

[3] D. Porath, A. Bezryadin, S. de Vries, and C. Dekker, Nature 403, 635 (2000).

[4] H. Y. Zhang and Y. J. Yan (unpublished).

[5] M. Büttiker, Phys. Rev. B 33, 3020 (1986); IBM J. Res. Develop. 32, 63 (1988).

[6] J. L. D’Amato and H. M. Pastawski, Phys. Rev. B 41, 7411 (1990).

[7] V. Mujica, M. Kemp, and M. A. Ratner, J. Chem. Phys. 101, 6849 (1994); ibid. 101, 6856 (1994).

[8] S. Datta, Electronic Transport in Mesoscopic Systems (Cambridge University Press, Cambridge, 1995).

[9] D. S. Fisher and P. A. Lee, Phys. Rev. B 23, 6851 (1981).

[10] X. Q. Li and Y. J. Yan, J. Chem. Phys. (submitted).

[11] S. Datta, W. Tian, S. Hong, R. Reifenberger, J. I. Henderson, and C. P. Kubiak, Phys. Rev. Lett. 79, 2530 (1997).

[12] See, for example, E. Meggers, M. E. Michel-Beyerle, and B. Giese, J. Am. Chem. Soc. 120, 12950 (1998); J. Jortner, M. Bixon, T. Langenbacher, and M. E. Michel-Beyerle, Proc. Natl. Acad. Sci. U.S.A. 95, 12759 (1998). 


\section{FIGURES}

FIG. 1. Schematic diagram for the electrical transport through DNA molecules. The DNA molecular wire consists of a stack of GC pairs, and each GC pair is connected with a fictitious electronic dephasing reservoir by a coupling strength $\eta$.

FIG. 2. I-V characteristics: theoretical results versus experimental data taken from Ref. [3]. The transport currents in the presence of weak dephasing $(\eta=0.05 \mathrm{eV})$ and stronger one $(\eta=0.3$ eV) are theoretically shown by the solid and dashesd curves.

FIG. 3. Dephasing effect on the differential conductance. The solid and dashed curves correspond to $\eta=0.05 \mathrm{eV}$ and $\eta=0.3 \mathrm{eV}$, respectively. The detailed change of the conductance profile is representatively shown in the inset. 
Fig. 1 by X.Q. Li et al: Electrical transport ...

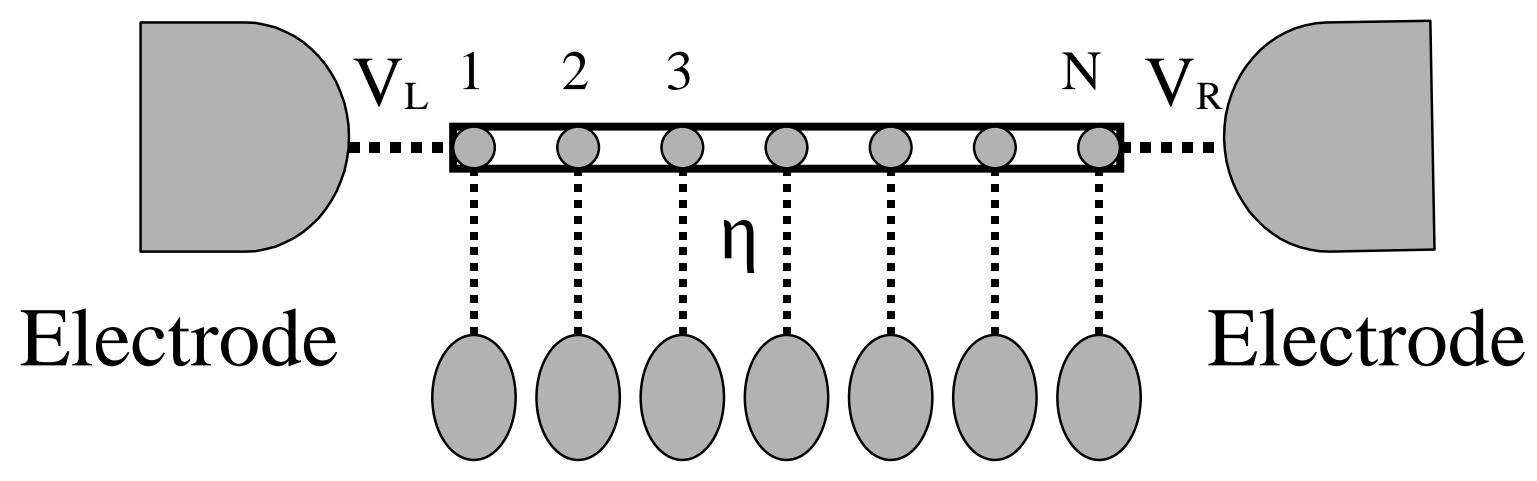

Dephasing reservoirs 
Fig. 2 by X.Q. Li et al: Electrical transport ...

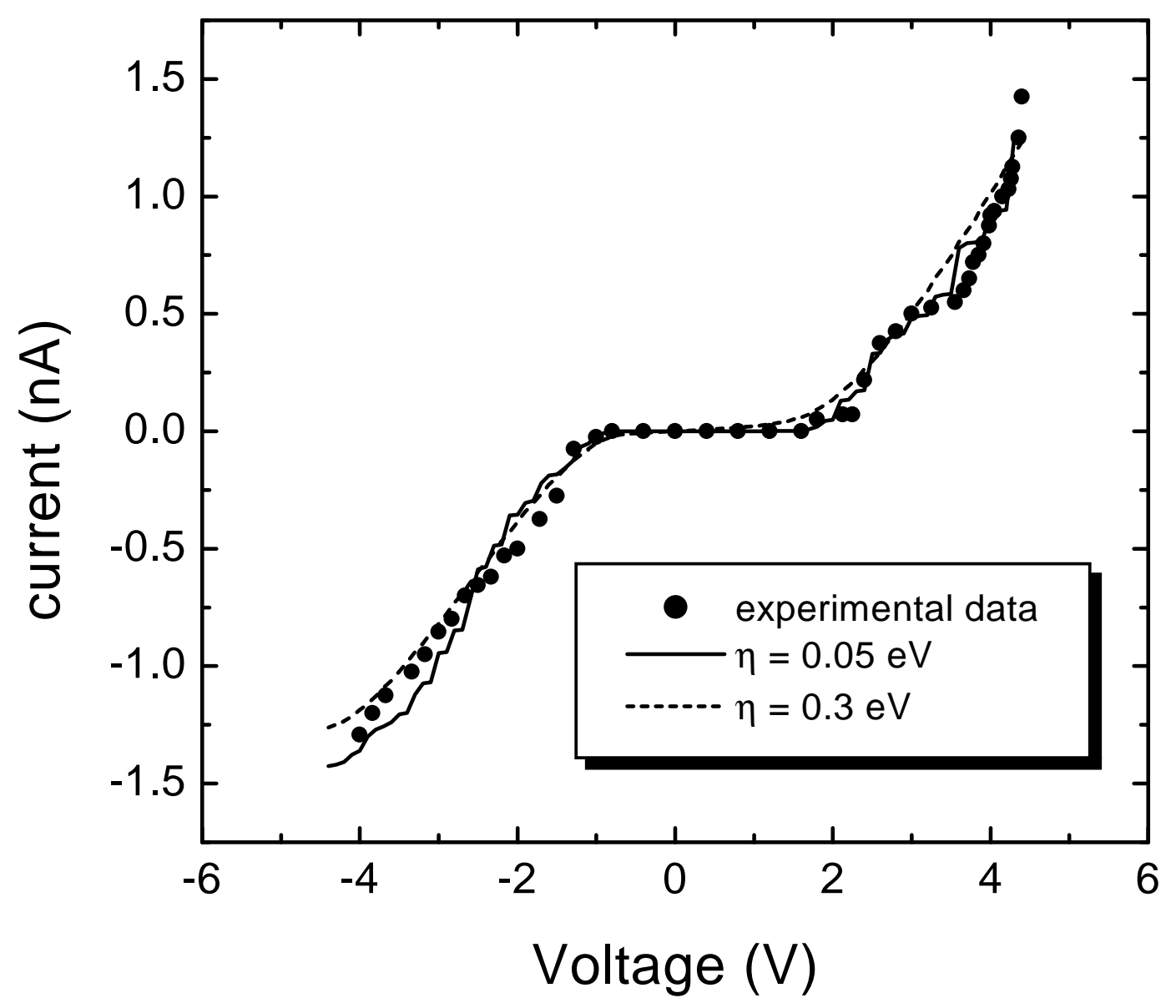


Fig. 3 by X.Q. Li et al: Electrical transport ...

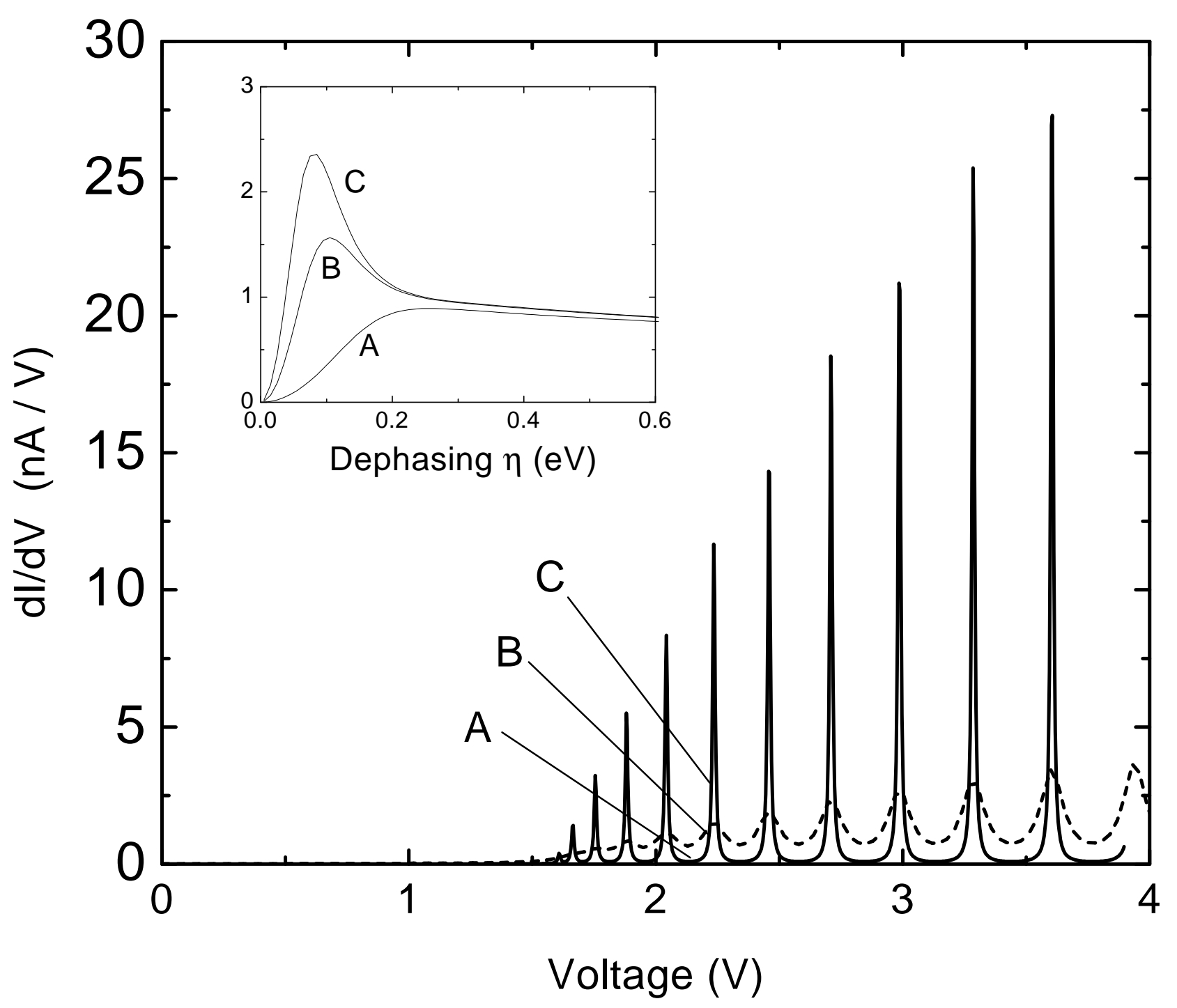

